\title{
PROPRIEDADE INSETICIDA DOS ÓLEOS ESSENCIAIS DE Piper hispidinervum C. DC.; Piper aduncum L. e Tanaecium nocturnum (Barb. Rodr.) Bur. \& K. Shum SOBRE Tenebrio molitor L., $1758^{(1)}$
}

\author{
Insecticidal properties of essential oils of Piper hispidinervum C. DC.; Piper aduncum L. and \\ Tanaecium nocturnum (Barb. Rodr.) Bur. \& K. Shum against Tenebrio molitor L., 1758.
}

\begin{abstract}
Murilo Fazolin ${ }^{2}$, Joelma Lima Vidal Estrela ${ }^{3}$, Valdomiro Catani ${ }^{4}$, Marcio Rodrigo Alécio ${ }^{5}$, Marilene Santos de Lima ${ }^{5}$
RESUMO

Óleos essenciais das piperáceas Piper aduncum L., Piper hispidinervum C. DC. e da bignoniácea Tanaecium nocturnum (Barb. Rodr.) Bur. \& K. Shum foram avaliados para o controle de larvas de Tenebrio molitor L., 1758. Para a avaliação do efeito por contato em superfície contaminada, foram utilizados papéis-filtro impregnados com diferentes quantidades dos óleos essenciais. Para a avaliação do efeito tópico foram aplicados $5 \mathrm{~mL}$ de soluções com diferentes concentrações dos óleos sobre larvas de quinto instar do inseto. A taxa de mortalidade foi a variável utilizada para avaliar os experimentos. Todos os óleos essenciais apresentaram efeito inseticida sobre larvas de T. molitor, sendo que as respostas variaram em função da concentração utilizada, assim como do método de exposição do inseto. A toxicidade dos óleos essenciais foi elevada apresentando os seguintes valores de $\mathrm{CL}_{50}: 0,045 ; 0,033 \mathrm{e} 1,515 \mathrm{~mL}$ de óleo $\mathrm{cm}^{-2}$ para exposição por contato (papel filtro) aos óleos de P. hispidinervum, P. aduncum e T. nocturnum, respectivamente. Para a aplicação tópica, os valores da $\mathrm{DL}_{50}$ foram de: 0,$000025 ; 0,009$ e $0,000015 \mathrm{~mL}$ de óleo $\mathrm{mg}$ de inseto ${ }^{-1}$ para os óleos essenciais de $P$. hispidinervum, $P$. aduncum e T. nocturnum, respectivamente. Resultados promissores para o emprego desses óleos essenciais como inseticidas foram obtidos utilizando-se concentrações acima de $3,0 \%\left(\mathrm{v} \mathrm{v}^{-1}\right)$ para $P$. hispidinervum e $2,5 \%\left(\mathrm{v} \mathrm{v}^{-1}\right)$ para $P$. aduncum e T. nocturnum.
\end{abstract}

Termos para indexação: Pimenta longa, dilapiol, safrol, mandelonitrila, inseticidas botânicos.

\section{ABSTRACT}

Essential oils from Piper aduncum L., Piper hispidinervum C. DC. (Piperaceae) and Tanaecium nocturnum (Barb. Rodr.) Bur.\& K. Shum (Bignoniaceae) were tested against Tenebrio molitor L., 1758 larvaes. Filter paper with different amounts of oils were employed for contact toxicity effects study. For topical effect study, aliquots of $5 \mathrm{~mL}$ of oils at different concentrations were applied on larvaes of the fifth instar. Mortality rate was used to evaluate the assays. All essential oils possessed insecticidal effect to $T$. molitor, but the responses varied according to oil concentration and method of exposure. The toxicities of essential oils to T. molitor were elevated, with $\mathrm{LC}_{50}$ of $0.045,0.033$ and $1.515 \mathrm{~mL}$ of the oil $/ \mathrm{cm}^{2}$ for contact (filter-paper) applications of the P. hispidinervum, $P$. aduncum and T. nocturnum oil, respectively. For topical application the values of the $\mathrm{LD}_{50}$ were: $0.000025,0.009$ and $0.000015 \mathrm{~mL}$ of the oil $/ \mathrm{mg}$ of the insect, for $P$. hispidinervum, $P$. aduncum and T. nocturnum oil, respectively. The most promising results for the use of such oils as insecticide were obtained using concentrations above $3,0 \%\left(\mathrm{v} \mathrm{v}^{-1}\right)$ for $P$. hispidinervum and $2,5 \%\left(\mathrm{v} \mathrm{v}^{-1}\right)$ for $P$. aduncum and T. nocturnum essential oils.

Index terms: Long piper, dillapiol, safrole, mandelonitrile, plant insecticide.

(Recebido para publicação em 3 de janeiro de 2005 e aprovado em 6 de junho de 2006)

\section{INTRODUÇÃo}

A diversidade da flora brasileira apresenta um imenso potencial para a produção de compostos secundários, que têm sido demandados continuamente pela indústria, nas últimas décadas, devido ao incremento da utilização de produtos naturais na agropecuária (PLETSCH \& SANT'ANA, 1995). Segundo estes autores, $16 \%$ das 500.000 espécies de plantas, que se estima existirem no mundo, encontram-se na floresta Amazônica. No entanto, a pesquisa de substâncias ativas derivadas de plantas no Brasil ainda é muito insipiente. Até o início da década de 80 foi estimado que menos de $1 \%$ das espécies da flora brasileira eram conhecidas quanto aos seus constituintes químicos (GOTTLIEB et al., 1981). Mesmo considerando ter havido incrementos significativos a partir desse percentual nas últimas duas décadas, há, evidentemente, uma grande lacuna de conhecimento da nossa flora a ser preenchida. Em decorrência disso, desenvolver ensaios, isolar, caracterizar e finalmente sintetizar ou biossintetizar compostos de interesse no controle de insetos torna-se um desafio constante (SHAPIRO, 1991).

${ }^{1}$ Trabalho financiado pelo CNPq.

2Pesquisador Doutor em Entomologia - Embrapa Acre - Rodovia BR-364, km 14 - Cx. P. 392 -69.901-180 - Rio Branco, AC - murilo@cpafac.embrapa.br ${ }^{3}$ Pesquisadora Visitante, Mestre em Entomologia- CNPq- DCR - Rodovia BR 364, km 14 - Cx. P. 392 - 69.901-180 - Rio Branco, AC

${ }^{4}$ Químico, Doutor em Química - Embrapa Acre - Rodovia BR-364, km 14 - Cx. P. 392 - 69.901-180 - Rio Branco, AC.

${ }^{5}$ Graduando do Curso de Agronomia, Bolsista CNPq/PIBIC - Universidade Federal doAcre - Cx. P. 500 - 69.915-900 - Rio Branco, AC 
Plantas, como organismos que coevoluem com insetos e outros microrganismos, são fontes naturais de substâncias inseticidas e antimicrobianas, já que as mesmas podem ser produzidas pelo vegetal em resposta a ataques de insetos e microrganismos, respectivamente. $\mathrm{O}$ uso de extratos de plantas inseticidas, inclusive os compostos aleloquímicos como os óleos essenciais, eram empregados no controle de insetos antes do advento das substâncias orgânicas sintéticas (REGNAULT-ROGER, 1997).

As espécies do gênero Piper são amplamente aplicadas na medicina popular em função das propriedades microbianas exibidas por seus constituintes. Piper hispidinervum C. DC.; vulgarmente conhecida como pimenta longa, é rica em safrol, componente químico aromático, que é empregado como matéria prima na manufatura de heliotropina, importante fixador de fragrâncias, e butóxido de piperonila, agente sinergístico de inseticidas naturais (PIMENTEL et al., 1998a). A bioatividade de safrol e isosafrol foi estudada também por Huang et al. (1999) em relação à Tribolium castaneum Herbst.; 1797 e Sitophilus Zeamais Mots.; 1865, o que permitiu evidenciar que houve efeito fumigante dessas substâncias sobre adultos de ambas espécies de pragas em concentrações a partir de $0,90 \mathrm{mg} \mathrm{cm}^{-2}$ para o safrol e $0,42 \mathrm{mg} \mathrm{cm}^{-2}$ para o isosafrol.

Dentre as piperáceas da Amazônia, a pimenta de macaco, Piper aduncum L., é uma excelente produtora de óleo essencial, o qual possui alto teor do éter fenílico dilapiol (MAIA et al., 1998). Bernard et al. (1995) compararam o extrato alcóolico de folhas de dezesseis espécies de Piper, apontando P. aduncum como a de maior atividade inseticida para larvas de segundo instar de Aedes atropalpus (Coquillett). Nesse ensaio, extrato aquoso de plantas frescas de $P$. aduncum aplicado diretamente na água, na concentração de 10 ppm, causaram a mortalidade de 50\% das larvas de segundo instar desse culicídeo. Já o dilapiol isolado e purificado, apresentou, nas mesmas condições experimentais, $92 \%$ de eficiência no controle das larvas na concentração de 1 ppm.

Fazolin et al. (2005) obtiveram resultados promissores utilizando o óleo essencial de $P$. aduncum no controle de Cerotoma tingomarianus Bechyné, importante desfolhador de leguminosas da Amazônia. Nesse estudo, foi avaliada a intoxicação por contato, sendo que por superfície contaminada o valor da concentração letal $\left(\mathrm{CL}_{50}\right)$ foi de $0,003 \mathrm{~mL}$ de óleo $\mathrm{cm}^{-2}$ e por aplicação tópica a dose letal $\left(\mathrm{DL}_{50}\right)$ foi de $0,002 \mathrm{~mL}$ de óleo $\mathrm{mg}$ de inseto ${ }^{-1}$.

A utilização rotineira de plantas bioativas por populações tradicionais na Amazônia aponta o cipó de
"Kangàrà Kanê", Tanaecium nocturnum (Barb. Rodr.) Bureau. \& K. Shum. (Bignoniaceae), como inseticida, uma vez que os índios Kayapós (Pará) utilizam-no para matar abelhas (KERR \& POSEY, 1991).

Devido à abundância de $P$. hispidinervum, $P$. aduncum e T. nocturnum na Região de Rio Branco (AC), aliado à infra-estrutura de destilação já instalada na região, para a obtenção dos óleos essenciais de pimentas longas, foi conduzida uma série de bioensaios com o objetivo de comparar o efeito inseticida dos óleos essenciais dessas plantas. Parra (1998) considera que em bioensaios desta natureza deveriam ser utilizados insetos alvo. Entretanto, considera também que devido à dificuldade de criação de muitas dessas espécies, é comum a utilização de outros insetos que não sejam alvo, mas facilmente criados em laboratório, destacando-se dentre eles Tenebrio molitor L.; 1758. Trata-se de um coleóptero da família Tenebrionidae cujas larvas destroem farinhas fubás, farelos, rações, macarrão, grãos quebrados ou anteriormente danificados. Desta forma, utilizou-se essa espécie como modelo, objetivando viabilizar a utilização desses óleos no manejo integrado de pragas em pequenas propriedades da Amazônia Ocidental.

\section{MATERIAL E MÉTODOS}

Os experimentos foram conduzidos no Laboratório de Entomologia da Embrapa Acre utilizando-se larvas de $5^{\circ}$ instar de T. molitor. As plantas de $P$. hispidinervum e $P$. aduncum foram coletadas de um banco de germoplasma e as plantas de T. nocturnum da reserva florestal da instituição.

\section{Obtenção e composição dos óleos essenciais}

Plantas adultas de P. aduncum e P. hispidinervum foram coletadas na Coleção de Germoplasma da Embrapa Acre, cortadas a 0,4 m do solo, separando-se somente as folhas para processamento. A massa vegetal foi submetida à secagem por seis dias em secador solar, até atingir de 20 a 30\% de umidade, sendo revolvida duas vezes ao dia para facilitar a aeração Pimentel et al. (1998b). Para extração do óleo de T. nocturnum, foram selecionadas plantas que apresentaram secções de caule de até $1 \mathrm{~cm}$ de diâmetro separando-se as folhas frescas. Em todos os casos colocaram-se $500 \mathrm{~g}$ de folhas e 2,0 L de água em balão de fundo redondo de 5L. Submeteram-se as misturas ao processo de hidrodestilação, utilizando-se aparelho tipo Clevenger dotado de equalizador de pressão, seguindo-se a metodologia de Innocentini (2000). Após a decantação de cada óleo e secagem com sulfato de magnésio anidro,

Ciênc. agrotec., Lavras, v. 31, n. 1, p. 113-120, jan./fev., 2007 
obteve-se rendimento dos óleos essenciais na faixa de $3,0 \%$ a 3,5\% para $P$. hispidinervum, de 2,0 a 2,2\% para $P$. aduncum e de $0,8 \%$ para $T$. nocturnum.

A análise cromatográfica dos óleos essenciais de $P$. aduncum e $P$. hispidinervum foram efetuadas em cromatógrafo a gás HP5890, equipado com coluna de sílica fundida Agilent HP5 (30 m x 0,32 mm d.i. x 0,25 mm de espessura do filme) tendo hélio como gás de arraste a $1 \mathrm{~mL}$ $\mathrm{min}^{-1}$. Empregou-se gradiente de temperatura de 80 a $140^{\circ} \mathrm{C}$ $\left(4{ }^{\circ} \mathrm{C} \mathrm{min}-1\right)$ e de 140 a $240{ }^{\circ} \mathrm{C}\left(20^{\circ} \mathrm{C} \mathrm{min}^{-1}\right)$. O injetor e detector (FID) foram mantidos a $280{ }^{\circ} \mathrm{C}$ e $300{ }^{\circ} \mathrm{C}$, respectivamente. As amostras foram preparadas a $1 \% \mathrm{em}$ diclorometano e injetadas ( $1 \mathrm{~mL}$ ) com divisão de fluxo de 1:100. A quantificação das substâncias deu-se por integração eletrônica dos sinais, sendo que o tempo de retenção e a porcentagem dos principais constituintes estão de acordo com amostras autênticas fornecidas por Bizzo et al. (2001) e Lopes et al. (2001).

A análise do óleo essencial de T. nocturnum foi realizada em cromatógrafo gasoso (VARIAN, modelo 3800) acoplado a espectrômetro de massas (VARIAN-SATURN 2000), equipado com coluna VARIAN Va-5 (30 m x 0,25 mm d.i. x $0,25 \mathrm{~mm}$ de espessura do filme) e detector de ionização de chamas (FID). O injetor foi mantido a $250{ }^{\circ} \mathrm{C}$, detector a $280{ }^{\circ} \mathrm{C}$ e gradiente de temperatura de 60 a $240{ }^{\circ} \mathrm{C}\left(5^{\circ} \mathrm{C} \mathrm{min}{ }^{-1}\right)$. Foram injetados $1 \mathrm{~mL}$ de solução 1:1000 (óleo:solvente) em diclorometano com divisão de fluxo de 1:100. A quantificação das substâncias deu-se por integração eletrônica dos sinais. As análises de cromatografia gasosa acoplada a espectrômetro de massas foram realizadas no mesmo aparelho com interface para CG-MS SATURN 2000. As condições de análises foram: hélio como gás de arraste com fluxo de $1 \mathrm{~mL} \mathrm{~min}^{-1}$, programação de 60 a $240{ }^{\circ} \mathrm{C}\left(5^{\circ} \mathrm{C} \mathrm{min}{ }^{-1}\right)$, mantendo o injetor e a interface a $280^{\circ} \mathrm{C}$. O espectrômetro de massas operou com ionização por impacto eletrônico de 70 eV e varredura de 50 a 700 unidades. Os componentes individuais foram identificados por comparação com os espectros de massas da biblioteca de espectros. As análises foram realizadas no Laboratório de Análises Química do Instituto Médico Legal de Rio Branco, AC.

Realizou-se uma análise complementar visando à quantificação do teor de ácido cianídrico das folhas de $T$. nocturnum, utilizando-se as metodologias de Bradbury et al. (1999) e Egan et al. (1998). Foram tomadas $100 \mathrm{mg}$ de folhas, que após maceradas em almofariz foram acondicionadas em um pote plástico de $50 \mathrm{~mL}$ contendo 1 $\mathrm{mL}$ de água e papel de filtro com uma solução tampão de pH 6. Colocou-se à borda do pote um papel de picrato sem que tocasse o líquido. $\mathrm{O}$ controle consistiu de um pote contendo a solução tampão, água e o papel de picrato. Deixaram-se os potes em repouso por 18 horas à temperatura ambiente. Após esse período, mergulharamse em 5,0 mL de água por $30 \mathrm{~min}$, separadamente, os papéis de picrato do pote das folhas meceradas e do controle. Em seguida realizaram-se as leituras de absorvância $(510 \mathrm{~nm})$ das soluções de picrato das folhas, corrigida pela leitura da solução controle, em espectrofotômetro B422 Micronal. $\mathrm{O}$ teor de HCN total (ppm) foi calculado pela seguinte equação: $\mathrm{HCN}$ total $(\mathrm{ppm})=396 \mathrm{x}$ absorbância $\mathrm{x} 100$ peso da $\operatorname{amostra}^{-1}(\mathrm{mg})$.

\section{Obtenção de Tenebrio molitor}

Os insetos utilizados nos experimentos foram provenientes da Embrapa Recursos Genéticos, sendo criados em condições de laboratório em recipientes plásticos de $30 \mathrm{~cm}$ x $15 \mathrm{~cm}$ x $3 \mathrm{~cm}$ em sala climatizada a $24 \pm$ $1{ }^{\circ} \mathrm{C}$ e umidade relativa de $70 \% \pm 4 \%$. A alimentação de larvas e adultos consistiu de uma mistura de farelo de trigo, farinha de mandioca, farinha de rosca e aveia na proporção de 3:1:1:1 em peso. As larvas foram separadas dos adultos e quando atingiram o $5^{\circ}$ instar foram utilizadas na experimentação.

\section{Curvas de concentração $x$ mortalidade}

Foram realizados testes preliminares com larvas de T. molitor, para obtenção de faixas de respostas, ou seja, intervalos de concentração dos óleos que ocasionaram mortalidade do inseto desde próximo de zero até próximo de $100 \%$. Cada faixa de resposta foi obtida a partir de $50 \mathrm{~mL}$ de cada óleo essencial submetidos a diluições seqüenciais em acetona p.a. até obter-se a concentração de $0,1 \%\left(\mathrm{v} \mathrm{v}^{-1}\right)$ de cada óleo. Dentro dessa ampla faixa de concentração foram obtidas faixas mais estreitas de respostas. $\mathrm{Na}$ avaliação do efeito de contato em superfície contaminada as concentrações variaram de: $2,0 \%$ a $10 \%\left(\mathrm{v} \mathrm{v}^{-1}\right)$ para o óleo essencial de $P$. hispidinervum de $1 \%$ a $7,5 \%\left(\mathrm{v} \mathrm{v}^{-1}\right)$ para $P$. aduncum e de $10^{-1} \%$ a $7,5 \%\left(\mathrm{v} \mathrm{v}^{-1}\right)$ para $T$. nocturnum. Nas avaliações do efeito de contato via aplicação tópica essa faixa de resposta foi de $10^{-1} \%$ a $5 \%\left(\mathrm{v} \mathrm{v}^{-1}\right)$ para o óleo essencial de $P$. hispidinervum e de $10^{-1} \%$ a $10 \%\left(\mathrm{v} \mathrm{v}^{-1}\right)$ para os óleos essenciais de P. aduncum e T. nocturnum.

Dentro desses intervalos, foram estabelecidas entre cinco e seis concentrações, além de um controle (acetona). Essas concentrações foram usadas nos bioensaios definitivos de curva de concentração-mortalidade e determinação da concentração e dose com probabilidade de causar $50 \%$ de mortalidade $\left(\mathrm{CL}_{50} \mathrm{eDL}_{50}\right)$ para cada óleo, seguindo-se a metodologia descrita por Finney (1971). 


\section{Bioensaios Definitivos}

\section{Contato em superfície contaminada (papel filtro)}

Bioensaios de concentração-mortalidade foram realizados utilizando-se larvas de T. molitor em placas de Petri contendo papeis filtro impregnados com $0,5 \mathrm{~mL}$ das seguintes concentrações dos óleos essenciais: $0,1 \%, 1,0 \%$, $2,5 \%, 5,0 \%$ e $7,5 \%\left(\mathrm{v} \mathrm{v}^{-1}\right)$ para $P$. aduncum e $T$. nocturnum; e $1,0 \%, 2,0 \%, 3,0 \%, 4,0 \%$, e $5 \%\left(\mathrm{v} \mathrm{v}^{-1}\right)$ para $P$. hispidinervum Depois da evaporação total do solvente, os papéis filtro foram colocados nas placas de Petri, que em seguida receberam 20 larvas de $T$. molitor.

\section{Contato por aplicação tópica}

Os bioensaios consistiram na aplicação tópica de $0,5 \mathrm{~mL}$ das seguintes concentrações dos óleos essenciais: $0,1 \%, 1,0 \%, 2,5 \%, 5,0 \%, 7,5 \%$, e $10,0 \%\left(\mathrm{v} \mathrm{v}^{-1}\right)$ para $P$. aduncum e T. nocturnum; e 2,0\%, 3,0\%, 4,0\%, 5,0\%, 6,0\%, $8,0 \%$, e $10,0 \%\left(\mathrm{v} \mathrm{v}^{-1}\right)$ para $P$. hispidinervum. A aplicação foi realizada na região protorácica de cada larva, com auxílio de uma microsseringa. Posteriormente, foram formados grupos de dez indivíduos em cada placa de Petri.

Tanto nos testes preliminares como nos bioensaios definitivos, utilizaram-se placas de Petri $(9,0 \mathrm{~cm} \times 1,5 \mathrm{~cm})$, mantidas em estufa encubadora para B.O.D. climatizada à temperatura de $25^{\circ} \mathrm{C} \pm 1{ }^{\circ} \mathrm{C}$, umidade relativa de $70 \pm 5 \%$ e fotofase de 12 horas, até o momento da avaliação da mortalidade dos insetos. O tempo de exposição aos óleos foi de 48 horas. Considerou-se a mortalidade cumulativa no período.

O delineamento experimental utilizado foi o inteiramente casualizado com quatro repetições, sendo cada bioensaio repetido três vezes, realizando-se uma análise conjunta dos resultados.

Em todos os bioensaios, os dados de mortalidade de concentração resposta foram submetidos à análise de Probit, utilizando-se o software SAS (SAS INSTITUTE, 1989). Depois de estimadas as $\mathrm{CL}_{50}$ e $\mathrm{DL}_{50}$ foram usadas como concentrações discriminatórias na detecção da toxicidade em relação a $T$. molitor.

Os valores de mortalidade foram corrigidos pela fórmula de Abbott (BUSVINE, 1971), transformando-se os valores em arc sen $\sqrt{\mathrm{x} / 100+0,5}$ para realização da análise de variância e comparação das médias pelo teste de Scott \& Knott (1974), utilizando o Sistema para Análise de Variância- SISVAR (FERREIRA, 2000).

\section{RESULTADOS E DISCUSSÕES}

A análise cromatográfica (FID) dos óleos essenciais apontou como componente majoritário o dilapiol $(73,97 \%)$ e safrol $(94,72 \%)$ para $P$. aduncum e $P$. hispidinervum, respectivamente.

A análise do óleo essencial de $T$. nocturnum por cromatografia gasosa apontou como componente principal o benzaldeído $(88,4 \%)$, concordando com o trabalho precedente de Gottlieb et al. (1981). Por meio da análise qualitativa do óleo essencial por cromatografia gasosa acoplada ao detector de massas, foi possível identificar o benzaldeído que apresenta íons com $\mathrm{m} \mathrm{z}^{-1} 106\left(76 \%, \mathrm{M}^{+}, \mathrm{C}_{7} \mathrm{H}_{6} \mathrm{O}\right), \mathrm{m} \mathrm{z}^{-1} 105$ $\left(100 \%, \mathrm{C}_{7} \mathrm{H}_{5} \mathrm{O}\right)$ e $\mathrm{m} \mathrm{z}^{-1} 77\left(30 \%, \mathrm{C}_{6} \mathrm{H}_{5}\right)$; e a a-(benziloxi)mandelonitrila em m z $\mathrm{m}^{-1} 237\left(100 \%, \mathrm{M}^{+}, \mathrm{C}_{15} \mathrm{H}_{11} \mathrm{NO}_{2}\right) ; \mathrm{m}$ $\mathrm{z}^{-1} 192\left(10 \%, \mathrm{M}^{++}-45\right) ; \mathrm{m} \mathrm{z}^{-1} 116\left(76 \%, \mathrm{M}^{+}-121 ; \mathrm{C}_{8} \mathrm{H}_{6} \mathrm{~N}\right)$; $\mathrm{mz}^{-1} 105\left(95 \%, \mathrm{M}^{+}-132 ; \mathrm{C}_{7} \mathrm{H}_{5} \mathrm{O}\right)$. Não foi possível confirmar a presença de mandelonitrila no óleo essencial de T. nocturnum, o que pode ter ocorrido devido a sua instabilidade nas condições de análise. No entanto, foi determinada uma concentração média de 636 ppm de ácido cianídrico em folhas recémcoletadas de T. nocturnum, sugerindo que seja este o provável responsável pelo princípio inseticida do óleo. Para Vetter (2000), é conhecido o fato de que plantas cianogênicas acumulam ácido cianídrico via glicosídeos cianogênicos. A indicação da presença da a-(benziloxi)-mandelonitrila e benzaldeído sugerem que o grupamento mandelonitrila poderá estar associado à glicosídeos cianogênicos, fonte de ácido cianídrico quantificado nas folhas de T. nocturnum. Esta hipótese está sendo objeto de investigação.

O óleo essencial de $P$. hispidinervum apresentou os maiores valores da inclinação das curvas nas duas vias de intoxicação testadas (Tabela1). As inclinações das curvas de concentração x mortalidade são parâmetros indicativos da ação tóxica dos óleos (FRAGOSO et al., 2002). Essa maior inclinação da curva de concentração $x$ mortalidade do óleo de $P$. hispidinervum indica que pequena variação na concentração ou dose desse óleo provoca grande mortalidade do inseto alvo, quando comparado aos óleos essenciais de $P$. aduncum e $T$. nocturnum.

Considerando-se as concentrações dos óleos essenciais que promoveram mortalidade acima de $80 \%$ de larvas de T. molitor expostas por contato em superfície contaminada, pode-se destacar: $T$. nocturnum a $2,5 \%, 5,0 \%$ e $7,5 \%\left(\mathrm{v} \mathrm{v}^{-1}\right)$, P. aduncum a $2,5 \%, 5,0 \%, 7,5 \%$ e $10,0 \%\left(\mathrm{v} \mathrm{v}^{-1}\right)$ e $P$. hispidinervum a $3,0 \%, 4,0 \%$ e $5,0 \%\left(\mathrm{v} \mathrm{v}^{-1}\right)$ (Figura 1). O óleo essencial de $T$. nocturnum nas concentrações de $5,0 \%$ e $7,5 \%$ $\left(\mathrm{v} \mathrm{v}^{-1}\right)$ foi letal para a totalidade dos insetos por essa via de intoxicação, diferindo significativamente da

Ciênc. agrotec., Lavras, v. 31, n. 1, p. 113-120, jan./fev., 2007 
mortalidade provocada pelas maiores concentrações do óleo de $P$. aduncum $\left(7,5 \%\right.$ e $\left.10,0 \%\left(\mathrm{v} \mathrm{v}^{-1}\right)\right)$ e de $P$. hispidinervum $\left(4,0 \%\right.$ e $\left.5,0 \%\left(\mathrm{v} \mathrm{v}^{-1}\right)\right)$ que não diferiram significativamente entre si.

Pela volatilidade apresentada pelo óleo essencial de Piper hispidinervum pode-se também atribuir os altos valores de mortalidade de T. molitor ao efeito conjunto contato e fumigação, uma vez que experimentos realizados por Huang et al. (1999) avaliando a mortalidade de larvas e adultos de Tribolium castaneum Herbest, 1797 submetidos a aplicações de diferentes concentrações de safrol, somente isolaram o efeito de contato por superfície contaminada quando as arenas de vidro utilizadas na experimentação não foram cobertas.

Na avaliação do efeito da aplicação tópica dos óleos essenciais as concentrações que promoveram mortalidade acima de $80 \%$ de larvas de T. molitor foram: T. nocturnum a 5,0\%; 7,5\% e 10,0\% ( $\left.\mathrm{v} \mathrm{v}^{-1}\right), P$. aduncum a 2,5\%, 5,0\%, 7,5\% e $10,0 \%\left(\mathrm{v} \mathrm{v}^{-1}\right)$ e P. hispidinervum a $8,0 \%$ e $10,0 \%\left(\mathrm{v} \mathrm{v}^{-1}\right)$ (Figura 2). O óleo essencial de $P$. aduncum nas concentrações de $7,5 \%\left(\mathrm{v} \mathrm{v} \mathrm{v}^{-1}\right)$ e $10,0 \%\left(\mathrm{v} \mathrm{v}^{-1}\right)$ foi letal para a totalidade dos insetos por essa via de intoxicação não diferindo significativamente da mortalidade provocada pelo óleo de $T$. nocturnum na concentração de $10 \%\left(\mathrm{v} \mathrm{v}^{-1}\right)$. Os valores de mortalidade desse grupo de óleos/concentrações diferiram significativamente do óleo de T. nocturnum a $5 \%\left(\mathrm{v} \mathrm{v}^{-1}\right)$, P. aduncum a $2,5 \%\left(\mathrm{v} \mathrm{v}^{-1}\right)$ e $P$. hispidinervum a $8,0 \%$ e $10,0 \%\left(\mathrm{v} \mathrm{v}^{-1}\right)$, não diferindo, entretanto significativamente entre si (Figura 3).

A alta toxicidade do óleo essencial de $P$. aduncum a larvas de T. molitor em concentrações acima de 2,5\% $\left(\mathrm{v} \mathrm{V}^{-1}\right)$ pode ser atribuída à ação sinergista do óleo de $P$. aduncum, como sugerido nos experimentos realizados por Bernard et al. (1995) e Mukerjee et al. (1979), os quais determinaram que o grupo metilenodioxifenil presente nas lignanas é uma característica das piperáceas, e são consideradas importantes inibidores de monooxigenases dependentes de citrocromo P450. Desta forma, o efeito inseticida do dilapiol pode estar relacionado à ação conjunta desta lignana e outros compostos bioativos minoritários na composição do óleo essencial, tais como sarisan (BIZZO et al., 2001) e principalmente, safrol (HUANG et al., 1999), uma vez que eles apresentam em sua estrutura o grupo metilenodioxifenil (Figura 3), sendo relatados como inseticidas naturais. 


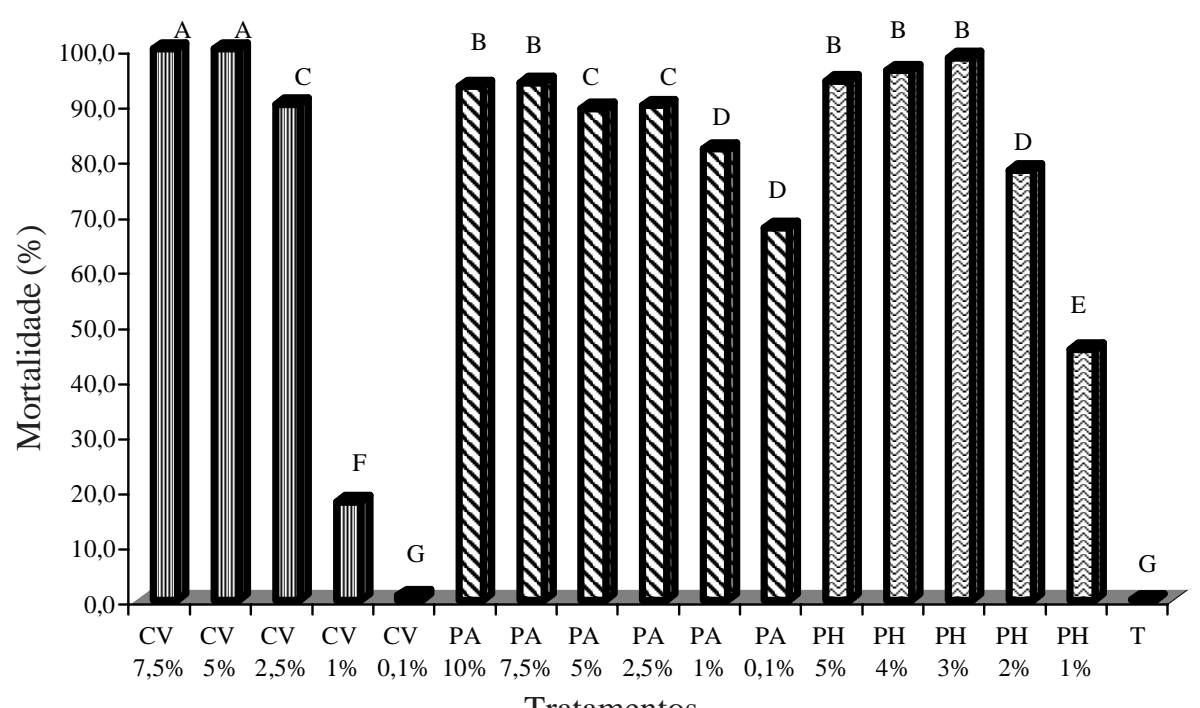

FIGURA 1 - Mortalidade de Tenebrio. molitor por diferentes concentrações de óleos essenciais de Tanaecium. nocturnum, Piper. aduncum e P. hispidinervum por via de contato em superfície contaminada.

Letras diferentes mostram diferenças entre as médias pelo teste de Scott-Knott a $\mathrm{P}<0,01$.

TN- Concentrações $\left(\mathrm{v} \mathrm{v}^{-1}\right)$ do óleo essencial de T. nocturnum; PA- Concentrações $\left(\mathrm{v} \mathrm{v}^{-1}\right)$ do óleo essencial de $P$. aduncum; $\mathrm{PH}-$ Concentrações $\left(\mathrm{v} \mathrm{v}^{-1}\right)$ do óleo essencial de $P$. hispidinervum e T- Testemunha.

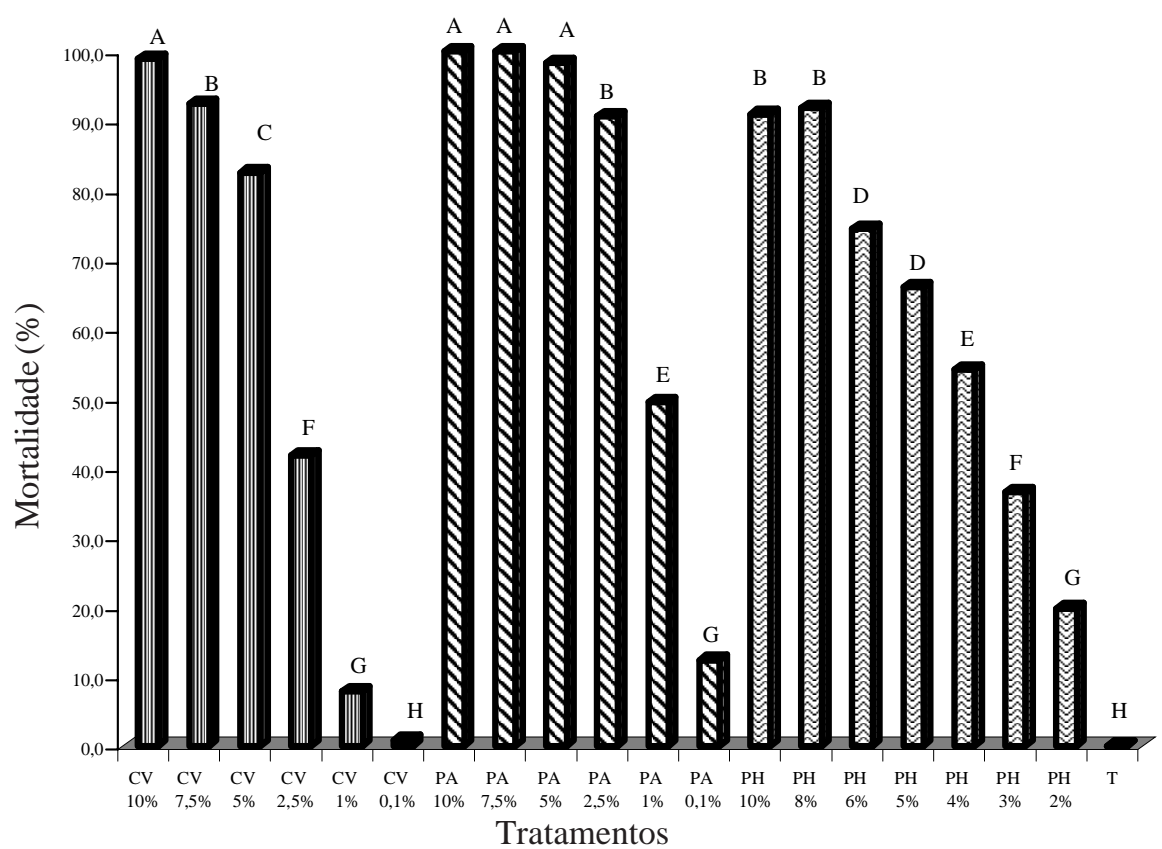

FIGURA 2 - Mortalidade de Tenebrio. molitor por diferentes concentrações de óleos essenciais de Tanaecium. nocturnum, Piper. aduncum e P. hispidinervum por via de contato por aplicação tópica. Letras diferentes mostram diferenças entre as médias pelo teste de Scott-Knott a $\mathrm{P}<0,01$.

TN- Concentrações $\left(\mathrm{v} \mathrm{v} \mathrm{v}^{-1}\right)$ do óleo essencial de T. nocturnum; PA- Concentrações $\left(\mathrm{v} \mathrm{v}^{-1}\right)$ do óleo essencial de $P$. aduncum; PH- Concentrações $\left(\mathrm{v} \mathrm{v}^{-1}\right)$ do óleo essencial de P. hispidinervum e T- Testemunha .

Ciênc. agrotec., Lavras, v. 31, n. 1, p. 113-120, jan./fev., 2007 


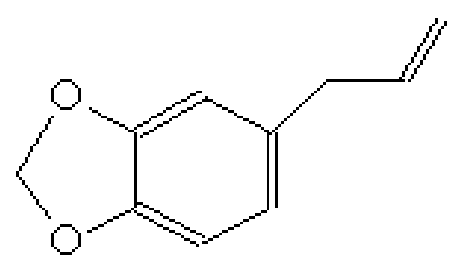

Safrol<smiles>C=CCc1cc2c(cc1OC)OCO2</smiles>

Sarisan<smiles>C=CCc1cc2c(c(OC)c1OC)OCO2</smiles>

Dilapiol

FIGURA 3 - Constituintes principais de Piper. hispidinervum e P. aduncum.

\section{CONCLUSÕES}

Os óleos essenciais de $P$. hispidinervum, $P$. aduncum e T. nocturnum foram tóxicos para larvas $T$. molitor variando os níveis de mortalidade em função da concentração e da via de intoxicação.

A utilização desses óleos essenciais pode ser considerada promissora como inseticida utilizando-se concentrações acima de $3,0 \%\left(\mathrm{v} \mathrm{v}^{-1}\right)$ de $P$. hispidinervum e $2,5 \%\left(\mathrm{v} \mathrm{v}^{-1}\right)$ de $P$. aduncum e T. nocturnum.

\section{AGRADECIMENTOS}

Os autores agradecem a participação dos técnicos Valdemir de Souza e Silva, Ronaldo Queiroz de Oliveira, Charles Rodrigues da Costa, Rubens Mamedio Bastos, John Lennon Mesquita Catão e Pedro Pereira da Silva.

\section{REFERÊNCIAS BIBLIOGRÁFICAS}

BERNARD, C. B.; KRISHINAMURTY, H. G.; CHAURET, D.; DURST, T.; PHILOGENE, B. J. R.; SANCHÉS-VINDAS, P.; HASBAUN, C.; POVEDA, L.; ROMAN, L. S.; ARNASON, J. T. Insecticidal defenses of piperaceae from the neotropics. Journal of Chemical Ecology, New York, v. 21, n. 6, p. 801-814, 1995.

BIZZO, H. R.; LOPES, D.; ABDALA, R. V.; PIMENTEL, F. A.; SOUZA, J.A. de; PEREIRA, M. V. G.; BERGTER, L.; GUIMARÃES, E. F. Sarisan from leaves of Piper hispidinervum C. DC (Long pepper). Flavour and Fragance Journal, Scotland, v. 16, p. 113-115, 2001.

BRADBURY, M. G; EGAN, S. V.; BRADBURY,J. H.Determination of all forms of cyanogens in cassava roots and cassava products using picrate paper kits. Journal of the Science and Food Agriculture, Washington, v. 79, p. 593-601, 1999.
BUSVINE, J. R. A critical review of the techniques for testing insecticides. 2. ed. London: Commonwealth Agricultural Bureaux, 1971. 345 p.

EGAN, S. V.; YEOH, H. H.; BRADBURY, J. H. Simple picrate paper kit for determination of the cyanogenic potential of cassava flour. Journal of the Science and Food Agriculture, Washington, v. 76, p. 39-48, 1998.

FAZOLIN, M.; ESTRELA, J. L. V.; CATANI, V.; LIMA, M. S.; ALECIO, M. R.

Toxidade do óleo de Piper aduncum L. a adultos de Cerotoma tingomarianus Bechyné (Coleoptera: Chrysomelidae). Neotropical Entomology, Vacaria, v. 34, n. 3, p. 485-489, 2005.

FERREIRA, D. F. Análise estatística por meio do SISVAR (Sistema para Análise de Variância) para Windows versão 4.0. In: REUNIÃO ANUAL DA REGIÃO BRASILEIRA DA SOCIEDADE INTERNACIONAL DE BIOMETRIA, 45., 2000, São Carlos. Anais... São Carlos: UFSCar, 2000. p. 255258.

FINNEY, D. J. Probit analysis. London: Cambridge University, $1971.33 \mathrm{p}$.

FRAGOSO, D. B.; JUSSELINO FILHO P.; PALLINI FILHO, A.; BADJI, C. A. Ação de inseticidas organofosforados utilizados no controle de Leucoptera coffeeella (GuérinMèneville) (Lepidóptera: Lyonetiidae) sobre o ácaro predador Iphiseiodes zuluagai Denmark \& Muma (Acari: Phytoseiidae). Neotropical Entomology, Londrina, v. 31, n. 3, p. 463-467, 2002. 
GOTTLIEB, O. R. M.; KOKETSU, M.; MAGALHÃES, M. T.; MAIA, J. G. S.; MENDES, P. H.; ROCHA, A. I.; SILVA, M. L.; WILBERG, V. C. Óleos essenciais da Amazônia VII. Acta Amazonica, Manaus, v. 11, p. 143-148, 1981.

HUANG, Y.; HO, S. H.; KINI, M. Bioactivities of safrole and isosafrole on Sitophilus zeamais (Coleoptera: Curculionidae) and Tribolium castaneum (Coleoptera: Tenebrionidae). Journal of Economic Entomology, Lanham, v. 92, n. 3, p. 676-683, 1999.

INNOCENTINI, A. P. Extração e caracterização analítica do óleo essencial da espécie Aniba canelilla, visando o isolamento do composto majoritário, 1-nitro-2-feniletano, e seu estudo toxicológico. 2000. 125 f. Dissertação (Mestrado em Química Analítica) - Universidade de São Paulo, São Carlos, 2000.

KERR, W. E.; POSEY, D. A. “Kangàrà Kanê” Tanaecium nocturnum (Bignoniaceae), um cipó usado pelos índios Kayapó como inseticida natural. Acta Amazonica, Manaus, v. 7, p. 23-26, 1991.

LOPES, D.; BIZZO, H. R.; OLIVEIRA, D. R.; LIMA, M. F; PIMENTEL, F. A. Avaliação química dos óleos essenciais de exemplares de pimenta longa (Piper hispidinervum $\mathrm{C}$. DC.) do Estado do Acre. Rio Branco: Embrapa-CPAF/AC, 2001. 226 p. (Documentos, 75).

MAIA, J. G. S.; ZOGHBI, M. G. S.; ANDRADE, E. H. A.; SANTOS, A. S.; SILVA, M. L.; LUZ, A. I. R.; BASTOS, C. N. Constituintes of the essential oil of Piper aduncum L. growing in the Amazon Region. Flavour and Fragrance Journal, Scotland, v. 13, p. 269-272, 1998.

MUKERJEE, S. K.; SAXENA, V. S.; TOMAR, S. S. New methylenedioxyphenyl synergists for pyrethrins. Journal of the Science and Food Agriculture, Washington, v. 27, p. 1209-1211, 1979.
PARRA, J. R. P. Criação de insetos para estudos com patógenos. In: ALVES, S. B. (Ed.). Controle microbiano de insetos. Piracicaba: FEALQ, 1998. 1163 p.

PIMENTEL, F.A., J.B.M. PEREIRA; M.N. DE OLIVEIRA. Zoneamento e caracterização de habitats naturais de pimenta longa (Piper hispidinervum) no Acre. Rio Branco: Embrapa CPAF/AC, 1998.17p. (Boletim de Pesquisa, 20).

PIMENTEL, F.A., W.G. CABRAL, M.R. DA SILVA; P.S.N. PINHEIRO. Processo de secagem de biomassa de pimenta longa (Piper hispidinervum). Rio Branco: Embrapa CPAF/ AC, 1998. 2p. (Comunicado Técnico, 98).

PLETSCH, M.; SANT'ANA, A. E. G. Secoundary compound accumulation in plants: the application of plant biotechnology to plant improvement. In: INTERNATIONAL SYMPOSIUM ON CHEMISTRY OF THE AMAZON, 2., 1995, Manaus. Anais... Manaus: Instituto Nacional de Pesquisas da Amazônia, 1995. v. 5, p. 51-64.

REGNAULT-ROGER, C. The potential of botanical essential oils for insects pest control. Integrated Pest Management Reviews, The Netherlands, v. 2, p. 25-34, 1997.

SAS INSTITUTE. SAS user's guide: statistics. Version 6.12. Cary, 1989.

SCOTT, A. J.; KNOTT, T. M. A cluster analysis method for grouping means in the analysis of variance. Biometrics, Washington, v. 30, p. 507-512, 1974.

SHAPIRO, J. P. Phytochemicals at the plant-insect interface. Archives of Insect Biochemistry and Physiology, London, v. 17, p. 191-200, 1991.

VETTER, J. Plant cyanogenic glycosides. Toxicon, Washington, v. 38, p. 11-36, 2000. 